\title{
A Rare Hemoglobinopathy as a Cause of Erythrocytosis in a Patient with Suspected JAK2V617F Negative Polycythemia Vera: A Case Report
}

\author{
Jaekel $\mathbf{N}^{1}$, Bauer $\mathrm{M}^{2}$, Behre $\mathrm{B}^{3}$ and Al-Ali HK ${ }^{4 *}$ \\ ${ }^{1}$ Department of Hematology and Oncology, University Hospital Halle, Germany, Ernst-Grube-Str. 40, Halle/Saale, 06120, Germany \\ ${ }^{2}$ Department of Pathology, University Hospital Halle, Germany, Magdeburger Str. 2, Halle/Saale, 06112, Germany \\ ${ }^{3}$ Amedes MVZ for pathology, cytodiagnostic and human genetics, Albert-Einstein-Straße 3, Halle/Saale, 06122, Germany \\ ${ }^{4}$ Krukenberg-Cancer-Center, University Hospital Halle, Germany, Ernst-Grube-Str. 40, Halle, 06120, Germany
}

\begin{abstract}
Introduction: Differential diagnosis of polyglobulia/erythrocytosis which is caused by deregulated erythropoiesis with an overproduction of red blood cells resulting in elevated hemoglobin and hematocrit levels is a diagnostic challenge.

Case Report: A 31-year-old man was referred to us with a suspected diagnosis of polycythemia vera (a clonal myeloproliferative neoplasm) because of erythrocytosis. One year before, he suffered from an ischemic central retinal vein occlusion of unknown cause. Aspirin treatment was initiated. His mother was diagnosed with a JAK2V617F negative polycythemia vera years earlier and treated with phlebotomies as well as aspirin. Apart from erythrocytosis, laboratory analysis showed a normal white blood cell and platelet counts. The differential blood picture and lactate dehydrogenase were within the normal ranges. Molecular testing for BCR-ABL, JAK2V617F and Calreticulin gene mutations by PCR was negative. Bone marrow biopsy was normal without signs for a myeloproliferative neoplasm. As almost all patients with polycythemia vera carry the phenotype-driver mutation JAK2V617F, further genetic testing for congenital causes of erythrocytosis was conducted. Mutations in the EPO-receptor gene were not found, but a very rare heterozygous point mutation in the beta-globin-chain [exon 2 (c.119A $>C$ ) leading to a change in codon $40(C A G>C C G)$ ] was detected by next generation sequencing. This rare variant belongs to the high oxygen affinity hemoglobinopathies leading to a reduction of oxygen supply in tissues and an increase in red cell production. Conclusion: The diagnosis of a JAK2 mutation negative polycythemia vera must always be questioned and congenital causes of erythrocytosis excluded as the therapeutic and prognostic consequences are immense. Rare variants of hemoglobinopathies, particularly those with high oxygen affinity need to be excluded by molecular testing.
\end{abstract}

Keywords: Polycythemia vera $•$ Polyglobulia $•$ High oxygen affinity variant haemoglobins

Abbreviations: BCR-ABL: Breakpoint Cluster Region-Abelson • Hb: Haemoglobin • Hct: Hematocrit • EPO: Erythropoietin • JAK2: Janus Kinase 2 $\cdot$ MPN: Myeloproliferative Neoplasm $\bullet \mathrm{O}_{2}$ : Oxygen $\cdot \mathrm{PV}$ : Polycythemia vera

\section{Introduction}

By definition, an absolute erythrocytosis is present when the red cell mass is greater than $125 \%$ of the predicted value for sex and body mass [1]. Nevertheless, an erythrocytosis is suspected when the hemoglobin $(\mathrm{Hb})$ and/ or hematocrit (Hct) is above the normal range, although a direct correlation between $\mathrm{Hb}$ and Hct measurement and red cell mass cannot be assumed [2].

A distinction is made between primary and secondary causes as well as acquired and congenital causes. An erythrocytosis is classified as primary when an intrinsic defect in the erythroid compartment of the bone marrow is present, for example in patients with congenital erythropoietin (EPO) receptor gene mutations and aquired polycythemia vera (PV). The secondary form is characterized by an overproduction of red cells in the bone marrow driven by endogenous and exogenous EPO or other reasons (e.g. Methemoglobinemia

*Address for Correspondence: Dr. Haifa Kathrin Al-Ali, Krukenberg-CancerCenter, University Hospital Halle, Germany, Ernst-Grube-Str. 40, Halle, 06120, Germany, E-mail: haifa.al-ali@uk-halle.de

Copyright: @ 2020 Jaekel N, et al. This is an open-access article distributed under the terms of the Creative Commons Attribution License, which permits unrestricted use, distribution, and reproduction in any medium, provided the original author and source are credited.

Received 28 March 2020; Accepted 01April 2020; Published 08 April 2020 or hypoxia). Measuring the EPO level helps to distinguish between primary and secondary causes [3].

According to the WHO-classification, the diagnosis of PV can be done by the presence of an elevated $\mathrm{Hb}$ level above $16.5 \mathrm{~g} / \mathrm{dl}$ in men and $16.0 \mathrm{~g} / \mathrm{dl}$ in women or a Hct above $49 \%$ in males or greater than $48 \%$ in females plus hypercellularity and panmyelosis in a bone marrow biopsy and a phenotypedriver mutation in JAK2, which can be detected in more than $95 \%$ of patients with PV $[4,5]$. The most frequent JAK2 mutation is JAK2V617F (exon 14) which is responsible for $97 \%$ of JAK2 mutations seen in PV. The remainder are spread across exons 12,13 , and 14 [6].

A correct work-up of erythrocytosis is sometimes a diagnostic challenge. Yet, a precise diagnosis is mandatory for accurate counselling of patients and initiating the optimal treatment.

\section{Case Report}

We present a case of a 31-year-old man referred to our center in September 2019 with a suspected PV. One year earlier, he suffered from an ischemic central retinal vein occlusion of unknown cause and was treated with intravitreal Bevacizumab and aspirin $100 \mathrm{mg}$ QD. Clinically, he complained of headache and visual impairment in both eyes. Social history was significant for smoking until 2015. The use of testosterone and steroids was denied. Cardiac-ultrasound showed normal results. At presentation, he was in a good 
general condition with a normal body mass index. Blood pressure and pulse were within normal ranges. Interestingly, his mother was diagnosed with a JAK2V617F negative PV some years before and was treated in an outpatient clinic with phlebotomies and aspirin. Furthermore, the patient stated that 13 years earlier, an elevated Hct was present in a routine blood examination.

Laboratory analysis showed normal white blood cell and platelet counts with 7.7 and $204 \times 10^{9} / \mathrm{L}$ respectively. $\mathrm{Hb}$ was $18.4 \mathrm{gm} / \mathrm{dL}$ with a $\mathrm{Hct}$ of $54 \%$ and an erythrocyte count of 6.25 million cells $/ \mathrm{mcL}$. The differential blood picture, lactate dehydrogenase, creatinine, albumin, C-reactive protein, as well as liver enzymes were within the normal ranges. Hepatosplenomegaly and lymphadenopathy were not detectable by physical examination and ultrasound. The EPO level was within the normal range $(11.1 \mathrm{U} / \mathrm{l})$ and the hemoglobinelectrophoresis showed no abnormalities (97.2\% HbA and 2.8\% $\mathrm{HbA} 2)$.

Molecular testing for BCR-ABL, JAK2V617F and Calreticulin gene mutations by PCR from the peripheral blood was negative. Furthermore, the histopathological examination of the bone marrow biopsy presented a mature hematopoiesis with predominant granulopoiesis and regular cellularity (Figure $1 \mathrm{a}, \mathrm{b})$. There was no apparent fibrosis and no atypia in the megakaryopoiesis (Figure 1c,d). Blast count in bone marrow smears was below 5\%. Cytogenetic analyses showed a normal karyotype. In summary, there were no signs for myeloproliferative neoplasia (MPN).

Because of the young age of the patient, the long history of polyglobulia, and the unusual JAK2 mutation negative polycythemia vera of his mother, we initiated genetic testing for an EPO-receptor mutation which yielded no pathological finding. A subsequent molecular testing by next generation sequencing for mutations, deletions, and duplications in the alpha-globin-genes $B P G M, E G L N, E P A S 1$ and $V H L$ was negative. Yet, a very rare heterozygous variant in the beta-globin-chain was detected. This variant was a point mutation in exon 2 (c.119A $>C$ ) leading to a change in codon 40 (CAG>CCG) resulting in a glutamine to proline replacement in the amino acid sequence of the beta globin chain. This variant is found in the $\alpha 1 \beta 2$ interface and belongs to the group of high oxygen $\left(\mathrm{O}_{2}\right)$ affinity hemoglobinopathies.

The symptoms of hyperviscosity (headache and visual impairment) disappeared under aspirin and phlebotomies. The target Hct is kept around $50 \%$. The patient and his family received an extensive genetic counselling because of the possible autosomal dominant inheritance of the disease. The subsequent molecular testing revealed the presence of the same mutation in his mother.

\section{Discussion}

Clarifying the cause of polyglobulia is sometimes a diagnostic challenge. A distinction must be made between primary and secondary as well as acquired and congenital causes [3]. First clues to the cause could often be found in the medical history of patients.

An increase in $\mathrm{Hb}$ levels, Hct, and the absolute number of red blood cells may be evidence of PV. However, more often erythrocytosis is caused by an underlying non-hematological disease. Reasons for an acquired secondary cause of polyglobulia such as pulmonary, renal, and cardiac causes have to be excluded first. Measuring the EPO-level could help differentiate between primary and secondary forms.

If there is no evidence for an acquired secondary cause or if there are additional features suggesting MPN such as abnormal white blood and/or platelet counts or splenomegaly, a hematological diagnostic work-up must be initiated including molecular testing for BCR-ABL and the typical phenotypedriver mutations for $B C R-A B L$ negative MPN as well as a bone marrow examination to confirm the diagnosis.

AccordingtotheWHO-classification of 2016, PVbelongstotheclassicalBCR$A B L$ negative MPN [4]. MPN are characterized by a persistent hyperactivation of the Janus kinase 2 (JAK2)-signal transducer and activator of transcription (STAT) signaling pathway which is driven by various somatic mutations.

In patients with a positive family history of polyglobulia, congenital causes should always be included in the differential diagnosis. Although most MPN cases are sporadic and occur in elderly adults, familial cases are described and there is a growing evidence for a germline predisposing factor in the MPN pathogenesis [7-9]. Therefore, the exclusion of a familial MPN should be part of the diagnostic work-up.

Yet, in the majority of patients with PV, a JAK2 mutation could be detected [7]. Thus, the diagnosis of a JAK2 mutation negative PV is very unusual and must always be questioned and congenital causes for erythrocytosis such as mutations in the EPO-receptor gene as well as mutations in the $\alpha$ - and $\beta$-globin-chains investigated [10]. Today and due to the improvement in molecular testing, congenital causes of erythrocytosis could be identified more easily [11]. One molecularly characterized form of congenital primary erythrocytosis is the autosomal dominant primary familial and congenital polycythemia caused by gain-of-function mutations in the EPOR gene which is found in around $12 \%$ of cases with erythrocytosis [12].

In 1966, Charache et al first described a molecular defect in the alphachain associated with an increased $\mathrm{O}_{2}$ affinity called $\mathrm{Hb}$-Chesapeake [13]. At present, more than $100 \mathrm{Hb}$-variants with high $\mathrm{O}_{2}$ affinity are described. Most of these $\mathrm{Hb}$ variants have substitutions at one of three regions that are crucial

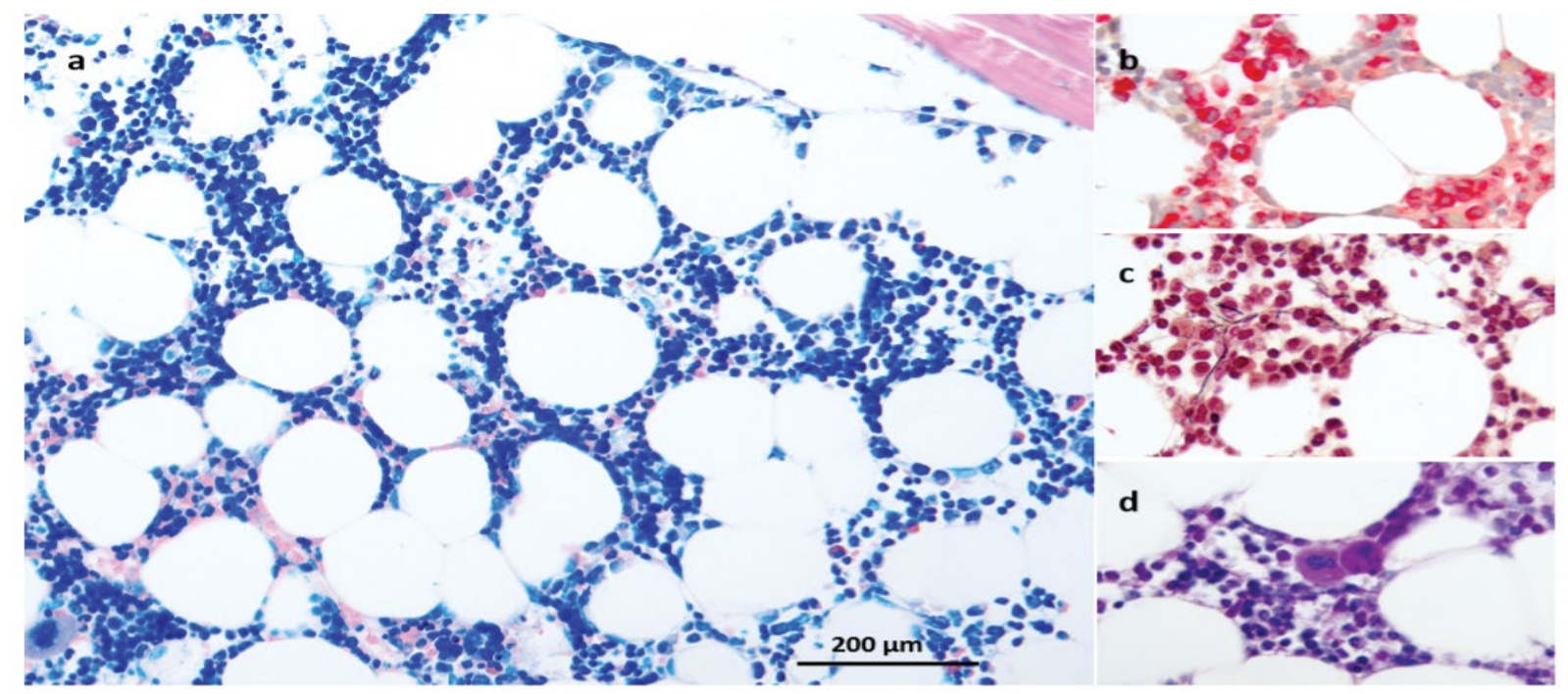

Figure 1: Histopathological examination of the bone marrow biopsy.

Giemsa staining; b) naphthol-AS-D-chloroacetate esterase with Visualization of the granulopoiesis; c) Gomori silver stain for fibrosis; d) Megakaryopoiesis in PAS reaction. 
for Hb function: 1 . the $\alpha 1 \beta 2$ interface; 2 . the $\mathrm{C}$-terminal end of the $\beta$-chain; and 3. the 2,3-bisphosphoglycerate mutase binding site [14]. The variant found in our patient is located at the $\alpha 1 \beta 2$ interface. To our knowledge, data to this variant are very limited.

Clinical data on patients suffering from congenital erythrocytosis are sparse. Patients are mostly asymptomatic and may have facial and mucosal erythrosis. Nevertheless, hyperviscosity symptoms and thromboembolic episodes have been reported and seem to be related to the high $\mathrm{Hct}$. The higher affinity of $\mathrm{O}_{2}$ to $\mathrm{Hb}$ leads to a lower oxygenation of tissues and consecutively to an erythrocytosis [10].

The primary goal of treatment is the prevention of thrombembolic complications and reduction of symptoms caused by hyperviscosity. Patients with thrombembolic events should be treated with antithrombotic agents as is the case in our patient. In patients with hyperviscosity, therapeutic phlebotomy could be indicated, targeting a Hct at which hyperviscosity symptoms are minimized, while ensuring that patients are not experiencing symptoms associated with reduced oxygen-carrying capacity. Basic measures include good hydration and the avoidance of activities that potentially increase blood viscosity (e.g., mountain climbing, smoking, dehydration) [15]

Guidelines on the investigation and management of erythrocytosis published in 2005 suggest that the target $\mathrm{Hct}$ with phlebotomy for patients with high affinity $\mathrm{Hb}$ should be $<60 \%$. If thrombosis or symptoms of hyperviscosity occur at lower Hct, then a target Hct of $52 \%$ is suggested [16]. It is important to note that the target Hct proposed here is higher than the $45 \%$ currently employed in patients with PV.

\section{Conclusion}

In conclusion, targeted NGS is cost-efficient, provides rapid and accurate mutation analysis, and could be applied directly to erythrocytosis cases where a genetic cause is suspected.

\section{Compliance}

Compliance with Ethical Standards.

\section{Conflict of Interest}

The authors declare that they have no conflict of interest.

\section{Informed Consent}

The patient has given his informed consent for each diagnostic and therapeutic step presented in this case report as part of the routine clinical work up.

\section{References}

1. Pearson, TC, DL Guthrie, J Simpson and S Chinn, et al. "Interpretation of Measured Red Cell Mass and Plasma Volume in Adults: Expert Panel on Radionuclides of the International Council for Standardization in Haematology". Br J Haematol 89 (1995): 748-756.

2. Johansson, Peter L, Soodabeh SafaißKutti and Jack Kutti. "An Elevated Venous Hemoglobin Concentration Cannot Be Used as a Surrogate Marker for Absolute Erythrocytosis: A Study of Patients with Polycythaemia Vera and Apparent Polycythaemia". Br J Haematol 129 (2005): 701-705.

3. McMullin, Mary Frances. "Investigation and Management of Erythrocytosis". Curr Hematol Malig Rep 11 (2016): 342-347.

4. Arber, Daniel A, Attilio Orazi, Robert Hasserjian and Jürgen Thiele, et al. "The 2016 Revision to the World Health Organization Classification of Myeloid Neoplasms and Acute Leukemia". Blood 127 (2016): 2391-2405.

5. Klampfl, Thorsten, Heinz Gisslinger, Ashot S Harutyunyan and Harini Nivarthi, et al. "Somatic Mutations of Calreticulin in Myeloproliferative Neoplasms". NEJM 19 (2013): 2379-2390

6. Alghasham, N, Y Alnouri, H Abalkhail and S Khalil. "Detection of Mutations in JAK2 Exons 12-15 by Sanger Sequencing". Int J Lab Hematol 38 (2016): 34-41.

7. James, Chloé, Valérie Ugo, Jean-Pierre Le Couédic and Judith Staerk, et al "A Unique Clonal JAK2 Mutation Leading to Constitutive Signalling Causes Polycythaemia Vera". Nature 434 (2005): 1144-1148.

8. Bellanné-Chantelot, Christine, Isabelle Chaumarel, Myriam Labopin and Florence Bellanger, et al. "Genetic and Clinical Implications of the Val617Phe JAK2 Mutation in 72 Families with Myeloproliferative Disorders". Blood 108 (2006): 346-352.

9. Tashi, Tsewang, Sabina Swierczek and Josef TPrchal. "Familial Mpn Predisposition”. Curr Hematol Malig Rep 12 (2017): 442-447.

10. Bento, C. "Genetic Basis of Congenital Erythrocytosis". Int J Lab Hem 40 (2018): 62-67.

11. Orvain, Corentin, Philippe Joly, Serge Pissard and Stéphanie Badiou, et al. "Diagnostic Approach to Hemoglobins with High Oxygen Affinity: Experience from France and Belgium and Review of the Literature". Ann Biol Clin 75 (2017): 39-51.

12. Huang, Lily J, Yu-Min Shen, Gamze B Bulut. "Advances in Understanding the Pathogenesis of Primary Familial and Congenital Polycythaemia". Br J Haematol 148 (2010): 844-852.

13. Charache, Samuel, David J Weatherall and John B Clegg. "Polycythemia Associated with a Hemoglobinopathy". J Clin Invest 45 (1966): 813-822.

14. Thom, Christopher S, Claire F Dickson, David A Gell and Mitchell J Weiss. "Hemoglobin Variants, Biochemical Properties and Clinical Correlates". Cold Spring Harb Perspect Med 3 (2013): a011858.

15. Bento, C, MF McMullin, Percy M and H Cario. "Primary Familial and Congenital Polycythemia". Gene Rev 2 (2016): 1-2.

16. McMullin, Mary MF, D Bareford, P Campbell and AR Green, et al. "Guidelines for the Diagnosis, Investigation and Management of Polycythaemia/Erythrocytosis". $\mathrm{Br}$ J Haematol 130 (2005): 174-195.

How to cite this article: Jaekel N, Bauer M, Behre B and Al-Ali HK, et al "A Rare Hemoglobinopathy as a Cause of Erythrocytosis in a Patient with Suspected JAK2V617F Negative Polycythemia Vera: A Case Report." J Mol Genet Med 14 (2020): 443 doi: $10.37421 / j m g m .2020 .14 .443$ 